\title{
A Comparison Analysis of Fog And Cloud Computing
}

\author{
${ }^{1}$ Gnaneswar Nair, ${ }^{1}$ Hadresh G, ${ }^{2}$ Pdinesh V \\ 1,2Lecturer, Information Technology Department, \\ ${ }^{1,2}$ R. C. Technical Institute, Ahmedabad, India
}

\begin{abstract}
In recent years, Fog Computing is introduced as a powerful extension of cloud computing and services to the edge of the network. Similar to Cloud, Fog provides data, compute, storage, and application services to end users. The fog computing is used to reduce the workload of the cloud computing by bringing basic computing services and analytic services to the edge of network, providing computing resources closer to the end devices, mobility support, improving overall network efficiency and performance and low latency. We explore the different applications of fog computing and various key comparison between cloud computing and fog computing.
\end{abstract}

\section{Index Terms - Fog Computing, Cloud Computing, IOT.}

\section{INTRODUCTION}

Cloud computing has emerged and got popularity by increasing development of storage and processing technology, as well as the success of the Internet, the cost-effectiveness of resource processing and availability. It has been increasingly adopted in many areas including science and engineering not to mention business due to its inherent flexibility, scalability and cost effectiveness [19]. Cloud computing is a very successful and effective technology, which is having many advantages like efficiency, cost effectiveness, easily accessible, backup and recovery, software automation and quick deployment etc. But still there are limitations like latency, security issues, issues related to internet connection due to increased ITO devices and smart devices now a days. Fog computing is a promising solution to deal with the demands of the ever-increasing number of Internet-connected devices. The idea of fog computing is to extend the cloud to be closer to the things that produce and act on IoT data. Instead of forcing all processing to back-end clouds, fog computing aims to process part of the services' workload locally on fog nodes, which are served as a near-end computing proxies between the front-end IoT devices and the back-end cloud servers. Putting resources at the edge of the network only one or two hops from the data sources allows fog nodes to perform low latency processing while latency-tolerant and large-scale tasks can still be efficiently processed by the cloud [2]. In addition, the cost and scale benefits of the cloud can help the fog to serve peak demands of IoT devices if the resources of fog nodes are not sufficient. Also, many applications require the interplay and cooperation between the edge (fog) and the core (cloud), particularly for the IoT and big data analysis [1]. From this point of view, fog computing is not aimed to replace cloud computing, but to complement it in a new computing paradigm, cloud-fog computing, which is to satisfy the increasingly sophisticated applications demanded by users [2]. Fog computing gives the cloud a companion to handle the two hexa bytes of data generated daily from the Internet of Things. Processing data closer to where it is produced and needed solves the challenges of exploding data volume, variety, and velocity [3].

\section{Cloud Computing Overview}

Simply put, cloud computing is the delivery of computing services, servers, storage, databases, networking, software, analytics, intelligence and more over the Internet ("the cloud") to offer faster innovation, flexible resources and economies of scale. You typically pay only for cloud services you use, helping lower your operating costs, run your infrastructure more efficiently and scale as your business needs change[20].

\section{Fog Computing Architecture}

In Cloud Computing architecture[21], it devided into two parts namely front-end and back-end. That which is visible to the clients and where there is an interface it called as the front end of Cloud Computing system. The cloud itself is the back end. It comes with all the networking, storage, computers, virtual systems that constitute the computing services. For every application there is a dedicated server which reflects the responsibility the CSP has for the clients [21]. From front end, users can use the cloud services from their devices. Clouds which are at backend and having servers, softwares or software platform, storages etc.

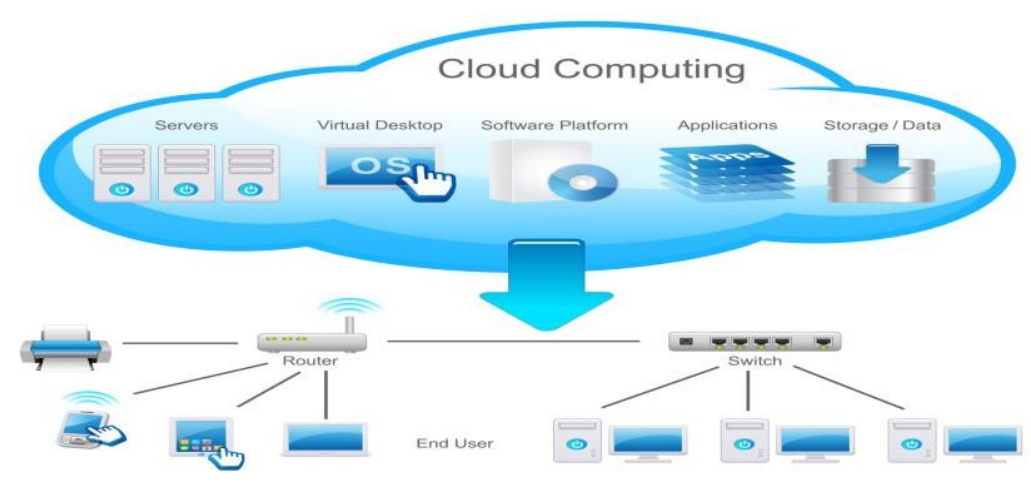

Fig. 1 The architecture of Cloud Computing ${ }^{[22]}$ 


\section{Advantages of cloud computing}

Cloud computing is a big shift from the traditional computing services. Here are some key advantages of it:

- Cost

Cloud computing eliminates the capital expense of infrastruscture which includes of buying hardware and software and setting up of it and the IT experts for managing the infrastructure. Use of cloud services is a cost effective approach.

\section{- Speed}

Most cloud computing services are provided self service and on demand, so even vast amounts of computing resources can be provisioned in minutes, typically with just a few mouse clicks, giving businesses a lot of flexibility and taking the pressure off capacity planning[20].

\section{- Global scale}

The benefits of cloud computing services include the ability to scale elastically. In cloud speak, that means delivering the right amount of IT resources - for example, more or less computing power, storage, bandwidth-right when it is needed and from the right geographic location[20].

\section{- Security}

Cloud computing offers great security when any sensitive data has been lost. Data can be easily accessed even something happens to your computer. It provides policies and technologies which can protect the data from potential threats.

\section{- Performance}

The biggest cloud computing services run on a worldwide network of secure datacenters, which are regularly upgraded to the latest generation of fast and efficient computing hardware. This offers several benefits over a single corporate datacenter, including reduced network latency for applications and greater economies of scale[20].

\section{- Availability}

Cloud services are available 24x7 from the providers. Most of the cloud providers are reliable and provides best services with maintaining good uptime. Services from cloud can be accessible from anywhere as it is having twenty four hours availability.

\section{Cloud Computing vs Traditional Computing}

Table 1. Difference between traditional computing and cloud computing [21]

\begin{tabular}{|c|c|c|}
\hline Parameters & Traditional computing & Cloud Computing services \\
\hline Pricing & $\begin{array}{l}\text { A firm would need huge upfront cost for both hardware } \\
\text { and software }\end{array}$ & Economical and predictable \\
\hline Security & $\begin{array}{l}\text { To ensure security the firm's IT experts should be better } \\
\text { than hackers }\end{array}$ & $\begin{array}{l}\text { Cloud services are regularly checked for any } \\
\text { security fault lines }\end{array}$ \\
\hline $\begin{array}{l}\text { Technical } \\
\text { support }\end{array}$ & $\begin{array}{l}\text { Contractual or per instance billing of any technical } \\
\text { glitches }\end{array}$ & $\begin{array}{l}\text { Unlimited technical support which comes within } \\
\text { the ambit of subscription fee }\end{array}$ \\
\hline Infrastructure & $\begin{array}{l}\text { Standalone server hardware and server software which is } \\
\text { pricey }\end{array}$ & $\begin{array}{l}\text { Multi-tenant systems shared by multiple cloud } \\
\text { customers }\end{array}$ \\
\hline Reliability & Depends on backup and in-house IT skills & $\begin{array}{l}\text { Professional technical expertise included within } \\
\text { the subscription fee }\end{array}$ \\
\hline Accountability & $\begin{array}{l}\text { After initial setup, provider is not typically bothered with } \\
\text { accountability }\end{array}$ & $\begin{array}{l}\text { The cloud provider can be held fully } \\
\text { accountable for any misgivings in the cloud } \\
\text { services }\end{array}$ \\
\hline
\end{tabular}

\section{Fog Computing OVERVIEW}

The term 'Fog computing' was proposed in 2012 by re- searchers from Cisco Systems [5]. The Fog is a distributed computing approach that mainly focuses on facilitating applications, which require low latency services [6], Fog computing also supports non- latency aware services. It is obvious that using idle computation resources near the users will improve overall service performance, if the volume of processing were not that high. A huge number of heterogeneous nodes will be connected to the Fog. These nodes include sensors and actuators among others [4]. Computation is performed in Fog devices when necessary and storage facilities are also available for a short period of time, at least in most Fog devices. Time-sensitive computation in the Fog is done without the involvement of third parties, and in most cases, is done by the Fog processing devices [7]. The most prominent characteristic of fog computing is the extension of the cloud service to the edge of network. It makes computation, communication, control and storage closer to end-users by pooling the local resources. The geographically distributed network edge devices consume data. Therefore, the data transfer time and the amount of network transmission is greatly reduced [8]. The fog paradigm can effectively meet the demands of real-time or latency-sensitive applications, and notably ease network bandwidth bottlenecks [9]. 


\section{Fog Computing Definition}

According to Cisco [3], the fog computing extends the cloud to be closer to the things that produce and act on IoT data. Also many researchers have defined Fog computing in different ways. Some of them are as follows:

- "Fog computing is a distributed computing platform where most of the processing will be done by virtualized and nonvirtualized end or edge devices. It is also associated with the cloud for non-latency-aware processing and long-term storage of useful data by residing in between users and the cloud". [7]

- "Fog computing is a paradigm with limited capabilities such as computing, storing and networking services in a distributed manner between different end devices and classic cloud computing. It provides a good solution for IoT applications that are latency-sensitive". [10]

- "Fog computing is a scenario where a huge number of heterogeneous (wireless and sometimes autonomous) ubiquitous and decentralized devices communicate and potentially cooperate among them and with the network to perform storage and processing tasks without the intervention of third parties. These tasks can be for supporting basic network functions or new services and applications that run in a sandboxed environment. Users leasing part of their devices to host these services get incentives for doing so." [5]

- "Fog computing is a highly virtualized platform that provides compute, storage, and networking services be- tween IoT devices and traditional cloud computing data centers, typically, but not exclusively located at the edge of network." [4]

- Fog Computing is a geographically distributed computing architecture with a resource pool which consists of one or more ubiquitously connected heterogeneous devices (including edge devices) at the edge of network and not exclusively seamlessly backed by Cloud services, to collaboratively provide elastic computation, storage and communication (and many other new services and tasks) in isolated environments to a large scale of clients in proximity". [11]

- "A scenario where a huge number of heterogeneous (wireless and sometimes autonomous) ubiquitous and decentralized devices communicate and potentially cooperate among them and with the network to perform storage and processing tasks without the intervention of third parties. These tasks can be for supporting basic network functions or new services and applications that run in a sandboxed environment. Users leasing part of their devices to host these services get incentives for doing so". [12]

\section{Fog Computing Architecture}

Fog computing architecture adds an extra resource-rich layer between end devices and cloud to meet these challenges in the low latency, high reliability and security, high performance, mobility, and interoperability [15][16]. The fog platform is composed of a large number of fog nodes. Fog nodes include various network edge devices and management systems within these devices, even some virtualized edge data centers [17]. According to [14], Fog computing is an approach that takes some of a data centre's operations to the edge of the network. The fog provides limited computing, storing and networking services in a distributed manner between end devices and the classic cloud computing data centres. The primary objective of fog computing is to provide low and predictable latency for time-sensitive IoT applications [13].

The reference model of fog computing architecture is a significant research topic. In these years, many researchers proposed a number of architectures for fog computing. They derived them mostly from the fundamental three-layer structure. Fog computing extends cloud service to the network edge by introducing fog layer between end devices and cloud. Fig. 1 shows the hierarchical architecture of fog computing [9].

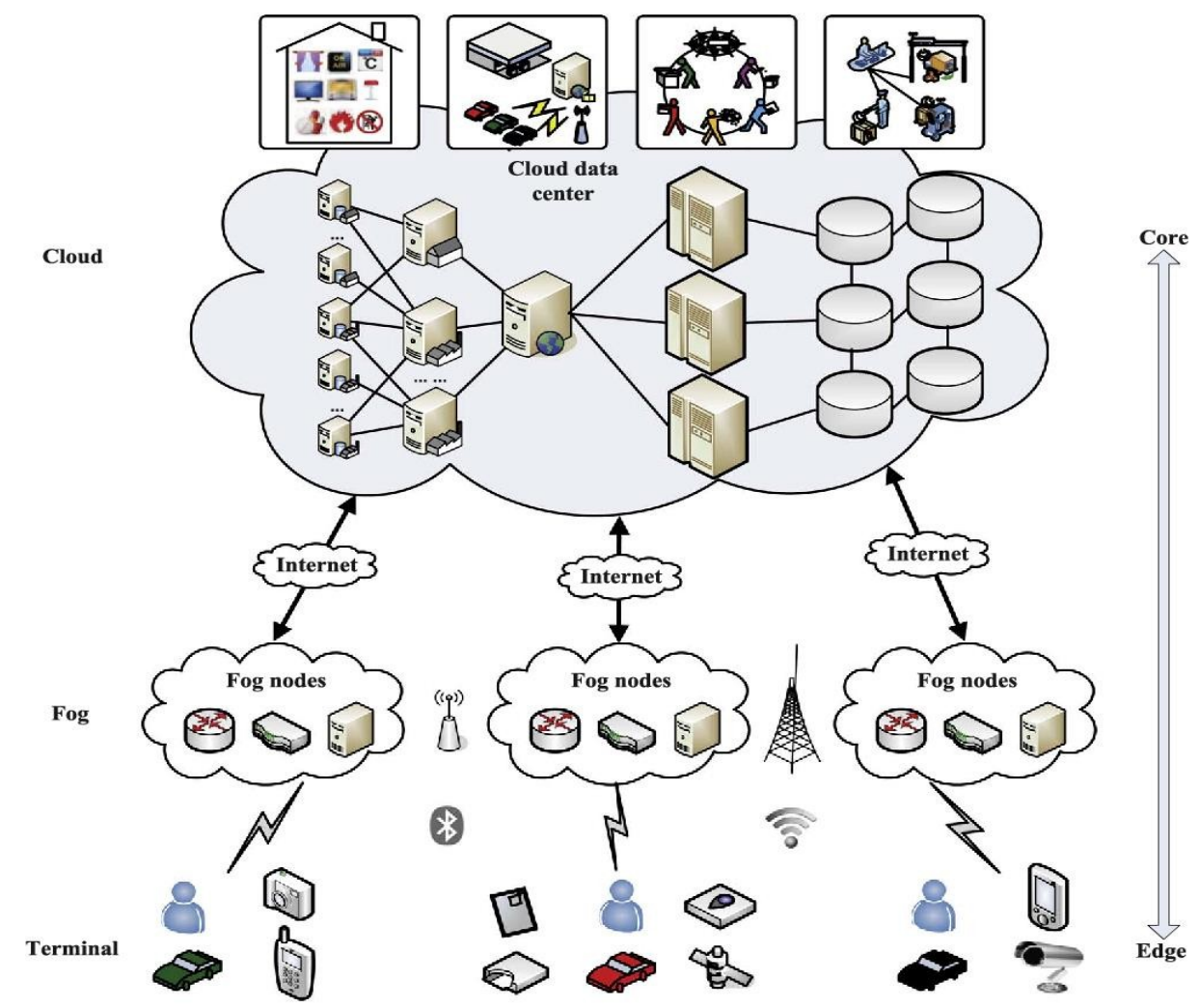

Fig. 1 The hierarchical architecture of Fog Computing ${ }^{[9]}$ 
The hierarchical architecture is composed of the following three layers:

Terminal layer: This is the layer closest to the end user and physical environment. It consists of various IoT devices, for example, sensors, mobile phones, smart vehicles, smart cards, readers, and so on. Specially, though the mobile phones and smart vehicles have the computing power, we only utilize them as the smart sensing devices here. These devices are widely geographically distributed in general. They are responsible for sensing the feature data of physical objects or events and transmitting these sensed data to upper layer for processing and storage [9].

Fog layer: This layer is located on the edge of the network. Fog computing layer is composed of a large number of fog nodes, which generally including routers, gateways, switchers, access points, base stations, specific fog servers, etc. These fog nodes are widely distributed between the end devices and cloud, for example, cafes, shopping centers, bus terminals, streets, parks, etc. They can be static at a fixed location, or mobile on a moving carrier. The end devices can conveniently connect with fog nodes to obtain services. They have the capabilities to compute, transmit and temporarily store the received sensed data. The real-time analysis and latency-sensitive applications can be accomplished in fog layer. Moreover, the fog nodes are also connected with cloud data center by IP core net- work, and responsible for interaction and cooperation with cloud to obtain more powerful computing and storage capabilities [9].

Cloud layer: The cloud-computing layer consists of multiple high-performance servers and storage devices, and provides various application services, such as smart home, smart transportation, smart factory, etc. It has powerful computing and storage capabilities to support for extensive computation analysis and permanently storage of an enormous amount of data. How- ever, different from traditional cloud computing architecture, not all computing and storage tasks go through the cloud. According to the demand-load, the cloud core modules are efficiently managed and scheduled by some control strategies to improve utilization of the cloud resources [18].

\section{Cloud vs Fog Computing}

Table 1 shows the technical differences between the cloud and the Fog. Surely we cannot say that the Fog is replacement of the Cloud. Definitely, it cannot be said that the Fog can replace the cloud. Also we cannot say that Fog is better than Cloud or Cloud is better than Fog as they both contribute differently by fulfilling different perspectives and requirements.

Table 1. Technical difference between Fog and cloud [9]

\begin{tabular}{|c|c|c|}
\hline Sr. No. & Cloud Computing & Fog Computing \\
\hline 1. & Cloud servers centralized and may far from end users. & Fog nodes are closer to the end users \\
\hline 2. & Cloud computing requires powerful server systems. & Fog nodes may be any devices with computing power. \\
\hline 3. & They are having centralized cloud servers. & Fog devices may be centralized or distributed. \\
\hline 4. & Storage and computation capacity are very high. & They are having low computing and storage capacity. \\
\hline 5. & Not mobile device, so it has very less mobility support. & Fog devices supports higher mobility. \\
\hline 6. & $\begin{array}{l}\text { Tasks execute on cloud have more latency as they are far } \\
\text { from client location. }\end{array}$ & $\begin{array}{l}\text { Tasks executing on fog nodes have lower latency, as } \\
\text { the nodes are closer to the end users. So fog computing } \\
\text { is used in latency-aware applications. }\end{array}$ \\
\hline 7. & Cost for computation, hardware and network are high. & Hardware and computation cost is very low. \\
\hline 8. & $\begin{array}{l}\text { Data processing done at the remote servers so not much } \\
\text { useful for real-time applications. }\end{array}$ & $\begin{array}{l}\text { Processing and storage done at the edge of the network } \\
\text { (closer to source), so very useful for real-time } \\
\text { applications. }\end{array}$ \\
\hline 9. & Higher computing capabilities and storage capacity. & $\begin{array}{l}\text { Lesser computing capabilities and storage capacity } \\
\text { then cloud. }\end{array}$ \\
\hline 10. & Clouds are less secure than fog. & $\begin{array}{l}\text { Fog is more secure then cloud as fog is having } \\
\text { distributed architecture. }\end{array}$ \\
\hline 11. & $\begin{array}{l}\text { Network issues and interruption may occur as its internet } \\
\text { based system and costumer may face downtime because of it. }\end{array}$ & $\begin{array}{l}\text { No downtime in fog computing which improves } \\
\text { quality of service for customers. }\end{array}$ \\
\hline
\end{tabular}

\section{Conclusion}

In this paper, we analyze the key differences between cloud and fog computing. Fog computing has many advantages over cloud computing like cost, latency, mobility, security etc. Fog computing is better in real-time applications which require low latency. We cannot say that the fog computing is replacement of cloud computing and fog cannot replace the cloud. Also we cannot say that the fog is always better than cloud as they both fulfill different requirements. Fog computing can be a middle level between end devices and remote cloud servers. It process real-time low latency computing and processing locally. So fog is an intelligent gateway, which offloads clouds enabling more efficient data storage, computing and analysis. 


\section{REFERENCES}

[1] F. Bonomi, R. Milito, J. Zhu, and S. Addepalli, "Fog Computing and Its Role in the Internet of Things," in Proceedings of ACM SIGOPS, pp. 8792, 2012.

[2] Xuan-Qui Pham, Eui-Nam Huh, "Towards task scheduling in a cloud-fog computing system", The 18th Asia-Pacific Network Operations and Management Symposium (APNOMS) 2016.

[3] Cisco Systems, "Fog Computing and the Internet of Things: Extend the Cloud to Where the Things Are," www.Cisco.Com, p. 6, 2016. [Online]. Available: http://www.cisco.com/c/dam/en \{_\}us/ solutions/trends/iot/docs/computing- overview.pdf.

[4] F. Bonomi, R. Milito, J. Zhu, and S. Addepalli, "Fog computing and its role in the internet of things," in Proceedings of the first edition of the MCC workshop on Mobile cloud computing. ACM, 2012, pp. 13-16.

[5] L. M. Vaquero and L. Rodero-Merino, "Finding your way in the fog: To- wards a comprehensive definition of fog computing," ACM SIGCOMM Computer Communication Review, vol. 44, no. 5, pp. 27-32, 2014.

[6] J. Li, T. Zhang, J. Jin, Y. Yang, D. Yuan, and L. Gao, "Latency estimation for fog-based internet of things," in Telecommunication Networks and Applications Conference (ITNAC), 2017 27th International. IEEE, 2017, pp. 1-6. is íp.

[7] R. Kumar Naha, S. Garg, Dimitrios Georgakopoulos, Prem Prakash Jayaraman, Longxiang Gao, Yong Xiang, Rajiv Ranjan "Fog Computing: Survey of Trends, Architectures, Requirements, and Research Directions", DOI: 10.1109/ACCESS.2018.2866491, IEEE Access.

[8] S. K. Datta, C. Bonnet, J. Haerri, Fog computing architecture to enable consumer centric internet of things services, in: International Symposium on Consumer Electronics, 2015, pp. 1-2.

[9] Pengfei Hu, Sahraoui Dhelim, Huansheng Ning, Tie Qiu, Survey on Fog Computing: Architecture, Key Technologies, Applications and Open Issues, Journal of Network and Computer Applications, http://dx.doi.org/10.1016/j.jnca.2017.09.002

[10] Verma, M.; Bhardwaj, N.; Yadav, A.K., Real Time Efficient Scheduling Algorithm for Load Balancing in Fog Computing Environment. Int. J. Inf. Technol. Comput. Sci. 2016, 8, 1-10.

[11] Tadapaneni, N. R. (2019). Role of Fog Computing in the Internet of Things. International Journal of Scientific Research and Engineering Trends.

[12] Vaquero, L.M.; Rodero-Merino, L. Finding Your Way in the Fog: Towards a Comprehensive Definition of Fog Computing. ACM SIGCOMM Comput. Commun. Rev. 2014, 44, 27-32.

[13] Shi, Y.; Ding, G.; Wang, H.; Roman, H.E.; Lu, S. The fog computing service for healthcare. In Proceedings of the 2015 2nd International Symposium on Future Information and Communication Technologies for Ubiquitous HealthCare (UbiHealthTech), Beijing, China, 28-30 May 2015; pp. 1-5.

[14] Atlam, H.F.; Walters, R.J.; Wills, G.B. Fog Computing and the Internet of Things: A Review. Big Data Cogn. Comput. 2018, 2, 10.

[15] S. Yi, C. Li, Q. Li, A survey of fog computing: concepts, applications and issues, in: The Workshop on Mobile Big Data, 2015, pp. 37-42.

[16] I. Stojmenovic, S. Wen, The fog computing paradigm: Scenarios and security issues, in: 2014 Federated Conference on

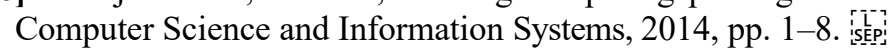

[17] H. Zhang, Y. Xiao, S. Bu, D. Niyato, R. Yu, Z. Han, Fog computing in multi-tier data center networks: A hierarchical game approach, in: 2016 IEEE International Conference on Communications (ICC), 2016, pp. 1-6.

[18] S. Sarkar, S. Misra, Theoretical modelling of fog computing: a green com- puting paradigm to support iot applications, IET Networks 5 (2) (2016) 23-29.

[19] Young Choon Lee, Chen Wang, Albert Y. Zomaya, Bing Bing Zhou "Profit-driven Service Request Scheduling in Clouds" 2010 10th IEEE/ACM International Conference on Cluster, Cloud and Grid Computing.

[20] https://azure.microsoft.com/en-in/overview/what-is-cloud-computing/

[21] https://intellipaat.com/blog/what-is-cloud-computing/

[22] Patil, A., \& Rekha, P. An Analysis Report on Green Cloud Current Trends and Future Research Challenges.

[23] Deepa.B, Srigayathri.S, Visalakshi.S, “A review on cloud computing”, International Journal of Trend in Research and Development, Volume 4(1), ISSN: 2394-9333, Jan-Feb2017, pp. 197-199.

[24] Yi, S.; Hao, Z.; Qin, Z.; Li, Q. Fog computing: Platform and applications. In Proceedings of the 3rd Workshop on Hot Topics in Web Systems and Technologies, HotWeb 2015, Washington, DC, USA, 24-25 October 2016; pp. 73-78.

[25] Schauhan, Cs. (2016). A Shift from Cloud Computing Model to Fog Computing. Journal of Applied Computing

[26] Liu, A., \& Yu, T. (2018). Overview of Cloud Storage. International Journal of Scientific \& Technology Research. 